EESTI NSV TEADUSTE AKADEEMIA TOIMETISED 1954. III kd., nr. I ИЗВЕСТИЯ АКАДЕМИИ НАУК ЭСТОНСКОИ ССР 1954. ТоМ III, № 1

\title{
К ОПРЕДЕЛЕНИЮ СОСТОЯНИЙ РАВНОВЕСИЯ ДЛИННЫХ ЦИЛИНДРИЧЕСКИХ ОБОЛОЧЕК ПРИ ОСЕСИММЕТРИЧНОЙ НАГРУЗКЕ
}

\author{
н. А. Алумяэ, \\ доктор технических наук
}

В этой работе выводятся дифференциальные уравнения для исследования послекритической стадии тонкостенных длинных цилиндрических оболочек вращения, находящихся под действием внешнего бокового давления и осевой нагрузки. Предполагается, что нормальные усилия безмоментного напряженного состояния от осевой нагрузки будут или соизмеримы с таковыми от боковой нагрузки, или же меньше последних.

Вводя в рассмотрение малую постоянную $h$ - отношение характеризующей величины толщины оболочки к радиусу кривизны срединной поверхности, - устанавливаем асимптотические свойства состояний равновесия после потери устойчивости безмоментного напряженного состояния при $h \rightarrow 0$. Вместе с тем устанавливается точность того или другого варианта разрешающих уравнений. В работе приводятся уравнения, с помощью которых может быть исследована послекритическая стадия длинных оболочек; точность этих уравнений не будет меньше, чем $\sqrt{h}$ по сравнению с единицей.

При определении критического значения безмоментного состояния длинных оболочек показывается, что интегралы соответствующих (линейных) уравнений распадаются на две независимые группы, так как одна группа из четырех интегралов не зависит от величины критической нагрузки. Эта группа интегралов описывает обыкновенный краевой эффект у торцов оболочки. Отмеченное обстоятельство значительно упрощает как определение критической нагрузки, так и исследование послекритической стадии: оно дает возможность рассматривать только вторую группу интегралов, которые определяются из системы более простых дифференциальных уравнений, чем исходные. Для интегралов этой системы указываются соответствующие краевые условия - по два условия на каждый торец.

При коротких оболочках дифференциальные уравнения, если требовать точность $\sqrt{h}$, не распадаются на независимые группы. Точнее, если отношение радиуса кривизны срединной поверхности к длине оболочки будет величиной порядка $h^{r}$, то короткими можно назвать в отмеченном выше смысле оболочки, для которых $r<0$, а длинными - те оболочки, для которых $0<r<0,5$. При $r \geqslant 0,75$ поведение оболочки не отличается от кругового кольца и такие оболочки можно назвать весьма длинными. 
Само собой разумеется, что исследование весьма длинных оболочек проще, чем оболочек длинных. Исследование послекритической стадии оболочек, находящихся на границе между короткими и длинными $(r=0)$, также проще, чем исследование коротких или длинных оболочек. Все сказанное, разумеется, относится только к рассматриваемой задаче цилиндрической оболочки.

1. Постановка задачи. Рассмотрим тонкостенную цилиндрическую оболочку вращения (трубу) с радиусом кривизны срединной поверхности $R$, толщиной $t$ и длиной $L$. Предположим, что на торцах оболочка соединена с диафрагмами, которые являются или $(a)$ жесткими в своей плоскости, но гибкими при изгибе из своей плоскости или (б) жесткими и в своей плоскости, и при изгибе из своей плоскости, но не препятствующими осевым поступательным смещениям.

Срединную поверхность оболочки отнесем к безразмерным координатам $\xi, \Theta$, причем линии $\xi=$ const суть направляющие, а линии $\Theta=$ const - образующие цилиндрической поверхности. Қвадрат длины линейного элемента $d s$ определяем формулой

$$
(d s)^{2}=R^{2}\left[(d \xi)^{2}+(d \Theta)^{2}\right] .
$$

Пусть толщина оболочки $t$ является некоторой функцией от $\xi$, т. е. $t=t(\xi)$, непрерывной вместе со своими производными и мало отличающейся от ее характеризующей величины $t_{0}=t\left(\xi_{0}\right)$. В дальнейшем возникнет вопрос об асимптотических свойствах состояния равновесия при стремлении отношения $h=t_{0} / R$ к нулю.

Пусть оболочка находится под действием внешнего гидростатического давления $p(\xi)=\sigma q(\xi)$ и осевой силы $P=2 \pi R \sigma V$, где $\sigma$ - параметр нагрузки; $q(\xi), V=$ const зависят от вида, а не от величины нагрузки. Предположим, что $q(\xi)$ будет непрерывная функция от $\xi$. В этом случае при достаточно малых значениях параметра нагрузки $\sigma$ напряженное состояние оболочки будет безмоментным (вплоть до узких зон вблизи торцов). Тангенциальные усилия $T_{\xi}, T_{\theta}$ этого состояния определяются формулами

$$
T_{\xi}=\sigma V, \quad T_{\theta}=-\sigma R q(\xi)
$$

Вблизи торцов оболочки к безмоментному состоянию прибавляется еще смешанное напряженное состояние, так называемый краевой эффект. Однако, как показывают исследования, влиянием краевого эффекта при изучении поведения оболочки в целом можно пренебречь, и мы, следуя оправдавшейся традиции, не будем его рассматривать. Поэтому осесимметричное безмоментное состояние можно назвать также и начальным состоянием равновесия оболочки.

При достаточно малых значениях $\sigma$ осесимметричное напряженное состояние (1.2) является единственно возможным состоянием равновесия; при достаточно больших значениях $\sigma$, как известно, существуют также и неосесимметричные состояния равновесия. Целью данной работы мы ставим разработку основных вариантов уравнений для определения этих неосесимметричных состояний равновесия с надлежащей оценкой точности предложенных уравнений.

Неосесимметричные состояния равновесия мы опишем относительно безмоментного осесимметричного состояния, существующего, или по крайней мере возможного (если состояние равновесия неустойчивое) при том же значении параметра нагрузки $\sigma$. При переходе от осесимметричного состояния в неосесимметричное состояние появляются изгибающие 
моменты $M_{\xi}, M_{\theta}$, крутящий момент $H$, сдвигающее усилие $S$, а нормальные усилия переходят из $T_{\xi}, T_{\theta}$ в $T_{\xi}+S_{\xi}, T_{\theta}+S_{\theta}$. Дефбрмация при этом переходе дается относительными удлинениями $\varepsilon_{\xi}, \varepsilon_{\theta}$, сдвигом $\gamma$ и параметрами изменения кривизны $x_{\xi}, x_{\theta}, \tau$.

Физические соотношения теории упругих оболочек устанавливают следующие зависимости:

$$
\begin{array}{lll}
\varepsilon_{\xi}=\bar{B}\left(S_{\xi}-\nu S_{\theta}\right), & \varepsilon_{\theta}=\bar{B}\left(S_{\theta}-\nu S_{\xi}\right), & \gamma=2(1+\nu) \overline{B S} \\
M_{\xi}=D\left(x_{\xi}+v x_{\theta}\right), & M_{\theta}=D\left(x_{\theta}+\nu x_{\xi}\right), \quad H=(1-\nu) D \tau,
\end{array}
$$

где

$$
\bar{B}=\frac{1}{E t}, \quad D=\frac{E t^{3}}{12\left(1-v^{2}\right)},
$$

причем $E$ - модуль Юнга, $v$ - коэффициент Пуассона.

Соотношения (1.3) и (1.4) - приближенные, но их погрешность остается в пределах, позволяющих получить результаты с достаточной точностью. В этом можно убедиться впоследствии, когда известны свойства деформированного и напряженного состояний, руководствуясь соображениями, приведенными в статье $\left({ }^{1}\right)$.

Введем еще некоторые предположения. Пусть $R / L=h^{r}$, где $r-$ некоторое положительное число. Предположим, что при каждом дифференцировании по $\xi$ функция $t$ (толщина оболочки) уменьшается в $h^{r}$ раз или даже больше, т. е. $d t / d \xi \leq h^{r} t$. Предположим далее, что нормальные усилия начального напряженного состояния от осевой нагрузки будут или соизмеримы с кольцевыми усилиями этого же состояния или же меньше, т. е. $\left|T_{\xi}\right| \lesssim\left|T_{\theta}\right|$.

2. Характер неосесимметричного состояния равновесия. Начнем с рассмотрения неосесимметричного состояния, бесконечно близкого к начальному, осесимметричному состоянию. Исследование этого состояния отличается простотой, так как в случае бесконечно малого смещения из начального состояния геометрические и статические соотношения выражаются линейными дифференциальными уравнениями. Имеем три уравнения совместности деформации:

$$
\begin{gathered}
\tau-x_{\theta}^{\prime}=0, \\
-x_{\xi}+\tau^{\prime}-\frac{1}{R}\left(\varepsilon_{\xi}-\gamma^{\prime}\right)=0, \\
x_{\xi}-\frac{1}{R}\left(\varepsilon_{\xi}+\varepsilon_{\theta}^{\prime \prime}-\gamma^{\prime \prime}\right)=0
\end{gathered}
$$

и три уравнения равновесия:

$$
\begin{gathered}
S_{\xi}^{\prime}+S^{\cdot}+T_{\theta}\left(\gamma^{\cdot}-\varepsilon_{\theta}^{\prime}\right)=0 \\
S^{\prime}+S_{\theta}^{\cdot}-\frac{1}{R}\left(M_{\theta}+2 H^{\prime}\right)+T_{\xi}\left(\gamma^{\prime}-\varepsilon_{\xi}\right)=0 \\
S_{\theta}-R\left(T_{\xi} x_{\xi}+T_{\theta} x_{\theta}\right)+\frac{1}{R}\left(M_{\xi}^{\prime \prime}+2 H^{\prime}+M_{\theta}\right)=0
\end{gathered}
$$

где штрихом и точкой обозначены частные производные

$$
(\ldots)^{\prime}=\frac{\partial}{\partial \xi}(\ldots), \quad(\ldots)^{*}=\frac{\partial}{\partial \theta}(\ldots) .
$$


K этим уравнениям присоединяются физические соотношения (1.3), (1.4). Вследствие замкнутости оболочки решение данной системы дифференциальных уравнений должно быть периодическим относительно координаты $\Theta$ и при $\xi=0, \xi=\mathrm{L} / R$, в соответствии с указанными в первом разделе работы краевыми закреплениями $(a)$ или (б), должно удовлетворять следующим краевым условиям:

$$
\varepsilon_{\theta}=0, \quad x_{\theta}=0, \quad S_{\xi}=0, \quad M_{\xi}=0
$$

при краевом закреплении $(a)$ и условиям

$$
\varepsilon_{\theta}=0, \quad x_{\theta}=0, \quad R \tau+\gamma=0, \quad \varepsilon_{\theta}^{\prime}-\gamma=0
$$

при краевом закреплении (б).

Практически интересным является то наименьшее, так называемое критическое значение параметра нагрузки, при котором существует нетривиальное решение системы (2.1) - (2.6), (1.3), (1.4), удовлетворяющее краевым условиям (2.7) или (2.8). Поэтому задача может быть ограничена отысканием того решения, которое приводит именно к критическому значению параметра нагрузки.

Характер исследуемого состояния равновесия дается, очевидно, оценками отдельных неизвестных величин и их частных производных по координатам $\xi$ и $\Theta$. Эти оценки не должны приводить к противоречию ни с исходными уравнениями $(2.1)-(2.6)$ при физических соотношениях (1.3) и (1.4), ни с краевыми условиями. Общий характер перехода от осесимметричного состояния в неосесимметричное сводится к тому, что деформация при этом переходе выражается в значительных изменениях кривизны срединной поверхности при незначительных относительных удлинениях и незначительном сдвиге $\left({ }^{6}\right)$. Отсюда можно вывести, что в уравнениях равновесия $(2.4)-(2.6)$ члены, содержащие начальные усилия $T \xi$ и $T_{\theta}$, имеют наибольшее относительное значение в уравнении (2.6). Более того, если предполагать, что $\left|T_{\xi}\right| \lesssim\left|T_{\theta}\right| \lesssim E t h$, то в уравнениях $(2.4),(2.5)$ можно этими членами даже пренебречь (с точностью до $h$ ). Из уравнения (2.6) следует, что критическая нагрузка будет тем меньше, чем меньше $S_{\theta}$ по сравнению с наибольшей из величин EtR $\varkappa_{\xi}, E t R \varkappa_{\theta}$. То обстоятельство, что данное уравнение при введенном предположении $T_{\xi} \sim T_{\theta}$ не предписывает, какая из величин $\varkappa_{\xi}, x_{\theta}$ должна быть больше, имеет существенное значение. Действительно, в таком случае можно предположить, что форма срединной поверхности после потери устойчивости осесимметричного состояния будет близка к форме срединной поверхности при изгибании последней

$$
\varkappa_{\theta}=f_{0}^{\prime \prime}+\xi f_{1}^{\prime \prime}, \quad \tau=f_{1}, \quad \varkappa_{\xi}=0,
$$

где $f_{0}, f_{1}$ - произвольные функции переменной $\theta$ с периодом $2 \pi$, а

$$
\varepsilon_{\xi}=\varepsilon_{\theta}=\gamma=0 \text {. }
$$

По физическим соотношениям $(1.3),(1.4)$ в этом случае $S_{\xi}, S, S_{t}$ также равняются нулю, но изгибающие и крутящий моменты отличны от нуля.

Однако данные выражениями $(2.9),(2.10)$ деформация и усилия не удовлетворяют ни краевым условиям $(2.7),(2.8)$, ни уравнениям равновесия (2.5). Поэтому деформация оболочки происходит все же 
с растяжением-сжатием срединной поверхности, и из выражений (2.9) уместно вывести лишь оценку

$$
x_{\theta} \gg x_{\xi} .
$$

Так как деформация оболочки происходит с растяжением-сжатием срединной поверхности, то для удовлетворения условий совместности деформации $(2.1)-(2.3)$ естественно появляется состояние деформации $\varepsilon_{\xi}$, $\varepsilon_{\theta}, \gamma$, близкое к самоуравновешивающему состоянию тангенциальных усилий $S_{\xi}, S, S_{\theta}$ :

$$
S_{\xi}=F_{\theta}^{*}+\xi F_{1}^{*}, \quad S=-F_{1}, \quad S_{\theta}=0,
$$

где $F_{0}, F_{1}$ - произвольные функции переменной $\theta$ с периодом $2 \pi$. Из выражений (2.12) выведем для дальнейшего только оценку

$$
S_{\xi} \gg S_{\theta} \text {. }
$$

Учитывая (2.13), можем представить условие (2.3) в форме

$$
x_{\xi}=\frac{1}{R}\left[\bar{B} S_{\xi}^{*} \cdot \nu\left(\bar{B} S_{\xi}\right)^{\prime \prime}-2(1+\nu)\left(\bar{B} S^{\prime}\right)^{\prime}\right]
$$

вполне аналогично получим с учетом соотношения (2.11) условие (2.6) в виде

$$
S_{\theta}=T_{\theta} R x_{\theta}-\frac{1}{R}\left[D \ddot{x_{\theta}}+\nu\left(D \varkappa_{\theta}\right)^{\prime \prime}+2(1-\nu)\left(D \tau^{\cdot}\right)^{\prime}\right]
$$

Заметим, далее, что условия (2.1), (2.4) тождественно удовлетворяются, если положить

$$
x_{\theta}=\frac{1}{R} \psi^{*}, \quad \tau=\frac{1}{R} \psi^{\prime}, \quad S_{\xi}=\frac{1}{\bar{B}} \varphi^{\prime}, \quad S=-\left(\frac{1}{\bar{B}} \varphi\right)^{\prime},
$$

где $\psi, \varphi$ - произвольные функции, и что поэтому систему $(2.1)-(2.6)$ можно выписать в более компактной форме

$$
\begin{gathered}
\psi^{\prime \prime}-(\varphi \cdot+\varphi)^{*}-2(1+\nu)\left\{\frac{1}{t}\left[t\left(\varphi^{*}+\varphi\right)\right]^{\prime}\right\}^{\prime}+\nu \varphi^{\prime \prime} \cdot=0 \\
\frac{1}{t}(t \varphi)^{\prime \prime}+\sigma g \psi^{*}+\lambda^{2}\left(\psi^{*}+\psi\right)^{*}+2(1-\nu) \frac{1}{t R^{2}}\left[t^{3}\left(\psi^{*}+\psi\right)^{\prime}\right]^{\prime}+ \\
+\frac{\nu}{t R^{2}}\left(t^{3} \psi^{*}\right)^{\prime \prime}=0
\end{gathered}
$$

где

$$
\sigma g=\sigma g(\xi)=-T_{\theta} \bar{B}, \quad \lambda^{2}=\frac{t^{2}}{12\left(1-v^{2}\right) R^{2}} .
$$

Предположим, что интегралы системы дифференциальных уравнений $(2.17),(2.18)$ при дифференцировании по $\xi$ уменьшаются в $h^{-q}$ раз, а при дифференцировании по $\Theta$ увеличиваются в $h^{-p}$ раз, где $q$ и $p-$ пока неизвестные положительные числа. Тогда из системы $(2.17),(2.18)$ следует, что $\sigma \mathrm{g}(\xi)$ будет иметь наименьшее значение, если $p=0,25-0,5 q$, при этом $\sigma g(\xi) \sim h^{1,5+q}, \varphi \sim \psi h$. Таким образом, чем больше $q$, тем меньше $\sigma g(\xi)$ и это оправдывает наше исходное положение о поведении интегралов при дифференцировании. Однако, так как оболочка замкнута, то $p$ не может быть меньше нуля и поэтому при $q>0,5 \mathrm{gg}$ имеет наименьшее возможное значение - оно будет соизмеримо с $h^{2}$. Указанные оценки сохраняют силу и при отрицательных значениях $q$ (тогда интегралы уже возрастают при дифференцировании по $\xi$ ), до тех пор, пока $-q \leqslant p$, т. е. пока $q \geqslant-0,5$. 
Пользуясь полученными оценками, легко убедиться, что интегралы системы $(2.17),(2.18)$ приводят $q \leqslant 0,5$ к следующим соотношениям:

$$
x_{\xi} \sim h^{0,5+q} x_{\theta}, \quad S_{\theta} \sim h^{0,5+q} S_{\xi} ;
$$

сравнение с соотношениями $(2.11),(2.13)$ показывает, что система $(2.17)$, (2.18) приводит к надежным результатам, если $h^{0,5+q} \ll 1$. Надо отметить, что при выполнении этого условия сама система $(2.17),(2.18)$ может быть значительно упрощена. А именно, с точностью до $h^{0,5+q}$ по сравнению с единицей можно в уравнениях $(2.17),(2.18)$ пренебречь некоторыми членами и представить эти уравнения в виде

$$
\begin{gathered}
\psi^{\prime \prime}-\varphi \cdot \cdot-\varphi^{*}=0, \\
\frac{1}{t}(t \varphi)^{\prime \prime}+\sigma g \psi^{*}+\lambda^{2}\left(\psi^{\cdots}+\psi^{*}\right)=0 .
\end{gathered}
$$

От исследования случая $q>0,5$ мы пока откажемся, так как к нему оценки (2.20) не применимы.

Все дальнейшее в данном разделе сводится к определению неизвестной пока величйны $q-$ в зависимости от длины оболочки и ее краевых закреплений у торцов.

Решение системы (2.1)-(2.6) при соблюдении физических соотношений (1.3), (1.4) должно удовлетворить четырем краевым условиям (2.7) или (2.8) на контурных кривых $\xi=0, \xi=L / R$. Однако решение системы $(2.21),(2.22)$ может удовлетворять только двум краевым условиям на каждой контурной кривой. Отсутствующие пока четыре интеграла описывают, очевидно, краевые эффекты у торцов оболочки. Не вникая в/подробности, отметим лишь, что они могут быть определены без затруднений по уравнениям линейной теории оболочек, если только $h^{0,5+q} \ll 1$ Произвольные постоянные интегралов краевого эффекта легко исключаются с помощью краевых условий как в случае (2.7), так и в случае (2.8). Таким путем прихөдим к краевым условиям для решения системы дифференциальных уравнений $(2.21),(2.22)$ : при краевом закреплении (a) оно должно удовлетворить условиям

и условиям

$$
\psi=0, \quad \varphi=0
$$

$$
\psi=0, \quad \psi^{\prime}=0
$$

при краевом закреплении (б). При выводе этих условий также было допущено, что $h^{0,5+q} \ll 1$.

Интегралы системы $(2.21),(2.22)$ при краевых условиях (2.23) или (2.24) не могут уменьшаться при дифференцировании по $\xi$ больше, чем в $L / R \sim h^{-t}$ раз. Но так как к наименьшему значению критической нагрузки ведет наибольшее значение $q$, то отсюда следует, что

$$
q=r .
$$

3. Исследование послекритической стадии. Линейными уравнениями $(2.1)-(2.6)$, физическими соотношениями $(1.3),(1.4)$ и краевыми условиями (2.7) или (2.8) определяются свойства неосесимметричного состояния равновесия при критическом значении начального (осесимметричного) состояния равновесия, а также и само критическое значение параметра нагрузки. Этими уравнениями нельзя определить неосесимметричные состояния, которые хотя и мало, но не бесконечно мало отличаются от начального состояния равновесия. Таким образом, этими уравнениями нельзя определить ни напряжений неосесимметричного состоя- 
ния, ни потенциальную энергию оболочки в зависимости от величины внешней нагрузки. Эти зависимости устанавливаются при помощи нелинейных соотношений, учитывающих конечность деформащии оболочки и описывающих равновесие оболочки в деформированном состоянии. Точные нелинейные соотношения здесь ради краткости не приводятся; подробнее о них см. в работах $(2,4)$.

Рассмотрим в дальнейшем неосесимметричные состояния, которые количественно мало отличаются от начального состояния. Другими словами, мы будем рассматривать некоторую начальную часть послекритической стадии оболочки при заданной внешней нагрузке. Это ограничение задачи допускает использование оценок, найденных во втором разделе данной работы, для вывода упрощенных соотношений из точных, с одновременной оценкой точности полученных соотношений. Полученные во втором разделе оценки сводятся при $r \leqslant 0,5$ к следующему:

$$
\begin{gathered}
x_{\xi} \sim x_{\theta} h^{0,5+r}, \quad \tau \sim x_{\theta} h^{0,25+0,5 r}, \\
S_{\theta} \sim S_{\xi} h^{0,5+r}, \quad S \sim S_{\xi} h^{0,25+0,5 r}, \\
S_{\xi} \sim E t R x_{\theta} h, \quad T_{\xi} \sim T_{\theta} \sim E t h^{1,5+r},
\end{gathered}
$$

причем функции $\varkappa_{\xi}, \varkappa_{\theta}, \tau, S_{\xi}, S_{\theta}, S$ при дифференцировании по уменьшаются в $h^{-r}$ раз, а по $\Theta$ - увеличиваются в $h^{-0,25+0,5 r}$ раз.

Если в точных уравнениях пренебречь нелинейными членами, которые будут порядка $x_{\theta} h^{0,5+r}$ по сравнению с главными линейными членами или же меньше, а также линейными членами порядка $h^{0,5+r}$ по сравнению с главными членами, то приходим к следующим соотношениям

$$
\begin{gathered}
\tau-x_{\theta}^{\prime}=0 \\
-x_{\xi}+\tau^{\prime}-\left(\frac{1}{R}-x_{\theta}\right) \bar{B} S_{\xi}=0 \\
\left(\frac{1}{R}-x_{\theta}\right) x_{\xi}+\tau^{2}-\frac{1}{R^{2}} \bar{B} S_{\xi}=0 \\
S_{\xi}^{\prime}+S=0 \\
S^{\prime}+S_{\theta}-\bar{B} S_{\xi} S_{\xi}-\left(\frac{1}{R}-x_{\theta}\right) D x_{\theta}=0 \\
\left(\frac{1}{R}-x_{\theta}\right) S_{\theta}-T_{\theta} x_{\theta}-x_{\xi} S_{\zeta}-2 \tau S+\frac{D}{R^{2}} x_{\theta}=0
\end{gathered}
$$

где для уменьшения количества неизвестных в условиях совместности деформации (3.3), (3.4) относительные удлинения выражены при помощи физических соотношений через усилия, а в условиях равновесия (3.6), (3.7) изгибающие моменты - через параметры изменения кривизны.

Вводя функции $\psi$ и $\varphi$, определяемые формулами $(2.16)$ и, кроме того, следующие новые функции $\kappa_{\xi}$ и $e_{\theta}$ :

$$
k_{\xi}=R x_{\xi}, \quad e_{\theta}=\bar{B} S_{\theta},
$$

получим систему из четырех уравнений

$$
\begin{aligned}
& -k_{\xi}+\psi^{\prime \prime}-\left(1-\psi^{*}\right) \varphi^{*}=0, \\
& \left(1-\psi^{*}\right) k_{\xi}+\left(\psi^{\prime}\right)^{2}-\varphi^{\cdots}=0,
\end{aligned}
$$




$$
\begin{gathered}
\frac{1}{t}(t \varphi)^{\prime \prime}+e_{\theta}-q^{\cdot} \varphi^{*}-i^{2}\left(1-\psi^{*}\right) \psi^{\cdot}=0, \\
\left(1-\psi^{\cdot}\right) e_{\theta}+\sigma g \psi \cdot-k_{\xi} \varphi^{\cdot}+\frac{2}{t}(t \varphi)^{\prime} \psi^{\prime}+i^{2} \psi^{\cdots}=0 .
\end{gathered}
$$

Обратимся к разбору краевых условий, сопутствующих системе (3.9) - (3.12). Установление этих условий не представляло бы затруднений, если бы мы имели основание определить краевой эффект по уравнениям линейной теории оболочек. В этом случае целесообразно применить метод исключения произвольных постоянных интегралов краевого эффекта из краевых условий $\left(^{5}\right)$; он был уже использован во втором разделе работы для вывода краевых условий к системе $(2.21),(2.22)$. Более того, при «линейном» краевом эффекте этот метод приводит к тем же условиям:

$$
\psi=0, \quad \varphi=0
$$

при краевом закреплении (a) и к условиям

$$
\psi=0, \quad \psi^{\prime}=0
$$

при краевом закреплении (б). В самом деле, общие свойства решений систем (3.9)-(3.12) и $(2.21),(2.22)$ не отличаются одно от другого, а краевые условия $(2.7),(2.8)$ имеют силу и для послекритической стадии. Поэтому вывод краевых условий к нелинейным уравнениям (3.9)-(3.12) аналоггиен выводу краевых условий к линеаризованным уравнениям $(2.21),(2.22)$ и приводит к этим же результатам.

Вместе с тем, нетрудно убедиться, что краевой эффект может быть при одном несущественном ограничении величины деформации вполне обоснованно определен по уравнениям линейной теории оболочек.

В практических целях достаточно ограничиться рассмотрением состояний, где нормальное смещение ш срединной поверхности при переходе оболочки от осесимметричного состояния в неосесимметричное будет порядка $h^{0,5}$ по сравнению с радиусом срединной поверхности. Так как $\frac{1}{R} w \cdot \psi$, то это значит, что ограничиваются рассмотрением некоторой начальной части послекритической стадии, определенной в зависимоети от длины оболочेки условием

$$
\psi \lesssim h^{0,25+0,5 r} .
$$

Предположим теперь, что краевой эффект может быть определен по уравнениям линейной теории оболочек, и установим порядок величины краевого эффекта посредством условий $(2.7),(2.8)$. Поступая так, находим, что при условии (3.15) нормальное перемещение краевого эффекта $\tilde{w}$ будет величиною порядка $R h^{1+r}$, как при краевом закреплении $(a)$, так и при (б).

Обращаясь к нелинейным уравнениям краевого эффекта (они не отличаются от нелинейных уравнений пологих оболочек $\left({ }^{3}\right)$, нетрудно убедиться, что краевой эффект может быть в рассматриваемом случае деформации (функции, описывающие эту деформацию, возрастают при дифференцировании по $\Theta$ в $h^{-0,25+0,5 r}$ раз) определен по линейной теории, если $\tilde{w}$ удовлетворяет условию $\tilde{w} \sim R h^{1+r}$ и $h^{0,5+r} \ll 1$. Таким образом, мы не пришли к противоречивому выводу и поэтому определение краевого эффекта по линейной теории обосновано. Вместе с тем обоснованы краевые условия (3.13), (3.14). 
При построении вышеизложенной упрощенной теории мы считали, что $h^{0.5+r}$ будет мало по сравнению с единицей. Отсюда следует, что если выведенным уравнениям и краевым условиям предписать точность $h^{0,5}$, то они применимы для определения критической нагрузки и исследования послекритической стадии оболочек, характеристическая величина длины $r$ которых удовлетворяет условию

$$
0 \leqslant r \leqslant 0,5 \text {. }
$$

Не следует, однако, думать, что при $r>0$ точность уравнений будет излишне высока, так как она превысит $h^{0,5}$. В самом деле, в уравнениях (3.9) - (3.12) нелинейные члены будут при условии (3.15) порядка $h^{r}$ по сравнению с линейными и для обеспечения точности $h^{0,5}$ линейные части уравнений (3.9) - (3.12) должны обладать точностью $h^{0,5+r}$.

Необходимо еще отметить, что в случае $r=0$ уравнения (3.9) - (3.12) могут быть упрощены. А именно, пользуясь вышеприведенными оценками (3.1), нетрудно убедиться, что эти уравнения можно с точностью $h^{0.5}$ написать в виде

$$
\begin{gathered}
-k_{\xi}+\psi^{\prime \prime}=0, \\
(1-\psi \cdot) k_{\xi}+\left(\psi^{\prime}\right)^{2}-\varphi^{\cdots}=0, \\
\frac{1}{t}\left(t \varphi^{\prime \prime}\right)^{\prime \prime}+e_{\theta}=0, \\
\left(1-\psi^{\prime}\right) e_{\theta}+\sigma g \psi \cdot-k_{\xi} \varphi^{\cdot}+\frac{2}{t}(\operatorname{tg} \varphi)^{\prime} \psi^{\prime}+\lambda^{2} \psi^{\cdots}=0,
\end{gathered}
$$

краевые же условия к этой системе даются попрежнему условиями (3.13), (3.14).

Те члены в уравнениях (3.9)-(3.12), которые в уравнениях (3.17) (3.20) отброшены, при увеличении длины оболочки возрастают как $h^{0,5-r}$ по сравнению с единицей. В случае $r=0,5$ в уравнениях (3.9)(3.12) все члены равноценны в смысле их асимптотических оценок *

4. Об исследовании весьма длинных оболочек. Начиная с определенного значения отношения $R / L$, когда показатель $r$ в равенстве $R / L=h^{r}$ превышает 0,5 , неосесимметричные состояния характеризуются тем, что описывающие эти состояния функции не изменяются при дифференцировании по $\Theta$. Поэтому при $r=0,5$ происходит нарушение закономерностей, установленных в предыдущих разделах работы для области $0 \leqslant r \leqslant 0,5$. Тем не менее, из полученных результатов можно вывести некоторые общие положения, экстраполируемые и на область $r>0,5$. K этим положениям относятся неравенства

$$
x_{\xi} \ll x_{\theta}, f^{\prime \prime} \ll f,
$$

где $f$ - любая функция, определяющая тем или иным путем неосесимметричные состояния равновесия. Опираясь на полученные результаты, естественно полагать, что оценка $f^{\prime \prime} \sim h^{2 r} f$ имеет место также и в области $r>0,5$.

* По поводу терминологии отметим, что оболочки, для которых $r=0$, можно на звать представителями оболочек средней длины, а оболочки, для которых $r=0,5-$ представителями длинных оболочек. Области же определения оболочек средней длины н длинных зависят от точности, предписанной уравнениям. Если уравнениям предписана точность $h^{0,5}$, то область оболочек средней длины стягивается в точку $r=0$, потому что при $r<0$ нужно пользоваться уравнениями коротких (или пологих, что то же самое) оболочек, а при $r>0-$ уравнениями длинных оболочек (3.9)-(3.12). 
При помощи неравенств (4.1) можем из уравнений $(2.1)-(2.6)$ вывести систему

$$
\begin{gathered}
\left(\varphi^{*}+\varphi\right)^{*}-\psi^{\prime \prime}+\nu \lambda^{2}\left(\psi^{*}+\psi\right)^{\cdots}+\sigma \nu g\left(\psi^{*}+\psi\right)^{*}=0, \\
\frac{1}{t}(t \varphi)^{\prime \prime}+\sigma g \psi^{*}+\lambda^{2}(\psi+\psi)^{*}=0,
\end{gathered}
$$

причем здесь отброшены члены порядка $h^{2 r}$ по сравнению с главными, сохраненными в системе членами.

Из уравнений $(4.2),(4.3)$ следуют оценки

$$
\begin{gathered}
\varphi \sim \psi h^{2 r} \text { при } 0,5<r \leqslant 1, \\
\varphi \sim \psi h^{2} \text { при } r>1 .
\end{gathered}
$$

Далее имеем в области $0,5<r \leqslant 1$

$$
\begin{gathered}
S_{\xi} \sim E t R x_{\theta} h^{2 r}, \quad S \sim E t R x_{\theta} h^{3 r}, \quad S_{\theta} \sim E t R x_{\theta} h^{2}, \\
x_{\xi} \sim x_{\theta} h^{2 r}, \quad \tau \sim x_{\theta} h^{r}
\end{gathered}
$$

и в области $r>1$

$$
\begin{gathered}
S_{\xi} \sim S_{\theta} \sim E t R x_{\theta} h^{2}, \quad S \sim E t R x_{\theta} h^{2+r}, \\
x_{\xi} \sim x_{\theta} h^{2}, \quad \tau \sim x_{\theta} h^{r} .
\end{gathered}
$$

Ограничимся опять-таки рассмотрением начальной части послекритической стадии, определенной неравенством $w \lesssim h^{0,5} R$ для нормального перемещения ш точек срединной поверхности. Это ограничение в области $r>0,5$ приводит к условию

$$
\psi \lesssim h^{0,5} .
$$

Так как главные нелинейные члены при введенном ограничении будут лишь порядка $h^{0,5}$ по сравнению с главными линейными членами, то для обеспечения точности $h^{0,5}$ можно отбросить из точных нелинейных уравнений только те линейные члены, которые будут порядка $h$ по сравнению с главными.

Учитывая это, с помощью оценок (4.5) найдем, что состояния равновесия в области $0,5<r<0,75$ определяются решением следующей системы уравнений:

$$
\begin{aligned}
& -k_{\dot{\xi}}+\psi^{\prime \prime}-\left(1-\psi^{*}\right)\left(\varphi^{*}-v e_{\theta}^{\cdot}\right)=0, \\
& \left(1-\psi^{*}\right) k_{\xi}+\left(\psi^{\prime}\right)^{2}-\varphi^{\cdots}+\nu e_{\theta}=0, \\
& \frac{1}{t}(t \varphi)^{\prime \prime}+e_{\theta}^{\cdot}-\varphi^{\cdot} \varphi^{*}-\lambda^{2}\left(1-\psi^{*}\right) \psi^{*}=0, \\
& (1-\psi \cdot) e_{\theta}+\sigma g \psi \cdot-k_{\xi} \varphi^{\cdot}+\frac{2}{t}(t \varphi)^{\prime} \psi^{\prime}+\lambda^{2} \psi \cdots=0,
\end{aligned}
$$

где функции $k_{\xi}, e_{\theta}$ даются формулами (3.8). Можно установить (на чем, однако, не будем останавливаться), что краевые условия к системе (4.8)-(4.11) совпадают с краевыми условиями к системе (3.9)-(3.12), т. е. при краевом закреплении $(a)$ решение системы должно удовлетворить на контурных кривых условиям

$$
\psi=0, \varphi=0,
$$

при краевом же закреплении (б) - условиям

$$
\psi=0, \psi^{\prime}=0 .
$$


Для области $0,75 \leqslant r \leqslant 1$ получим систему уравнений, из которых первые два - условия совместности деформации - совпадают с уравнениями $(4.8)$, (4.9), но остальные - условия равновесия - будут значительно проще соответствующих уравнений $(4.10),(4.11)$ :

$$
\begin{gathered}
e_{\theta}^{\cdot}-\lambda^{2}\left(1-\psi^{\cdot}\right) \psi^{*}=0, \\
\left(1-\psi^{\cdot}\right) e_{\theta}+\sigma g \psi^{\cdot}+\lambda^{2} \psi^{\cdots}=0 .
\end{gathered}
$$

По своей структуре уравнения (4.14), (4.15) не отличаются от уравнений равновесия кругового кольца, находящегося под действием внешнего, нормального к кольцу давления.

Решение системы (4.14), (4.15) дает определенное представление о поведении весьма длинной оболочки после потери устойчивости осесимметричного состояния равновесия. Можно ограничиться интегрированием только этой системы, так как, во-первых, напряжения от тангенциальных усилий при $r \geqslant 0,75$ будут порядка $h^{0,5}$ или меньше по сравнению с напряжениями от изгиба $x_{\theta}$ срединной поверхности и поэтому определение функций $\varphi, k \xi$ интереса не представляет. Во-вторых, при $r=1$ условия совместности деформации (4.8), (4.9) становятся ненадежными, потому что осевые усилия будут столь малы по сравнению с изгибающими моментами, что определение удлинений и сдвига через усилия при помощи физических соотношений (1.3) будет незаконным.

\footnotetext{
Ннститут строительства и строительных материалов Академии наук Эстонской ССР
}

Поступила в редакцию $16 \times 1953$

\section{ЛИТЕРАТУРА}

1. Н. А. Алумяэ, О критическом значении осесимметричного безмоментного напряженного состояния длинной катеноидной оболочки, Прикл. мат. мех., т. XVI, вып. 6, 1952.

2. Н. А. А л у м я э, Дифференциальные уравнения состояний равновесия тонкостенных упругих оболочек в послекритической стадии, Прикл, мат. мех., т. XIII, вып. 1 . 1949.

3. В. З. В ла со в, Общая теория оболочек, Гостехиздат, М.-Л., 1949.

4. К. 3. Г а л и мов, К общей теории пластин и оболочек при конечных перемещениях и деформациях, Прикл. мат. мех., т. XV, вып. 6, 1951.

5. А. Л. Гольден в ей зе р, Некоторые приемы интегрирования уравнений теорин тонких оболочек, Прикл. мат. мех., т. Х, вып. 3, 1946.

6. С. П. Т и мошен ко, Устойчивость упругих систем, Гостехиздат, М.-Л, 1946. 